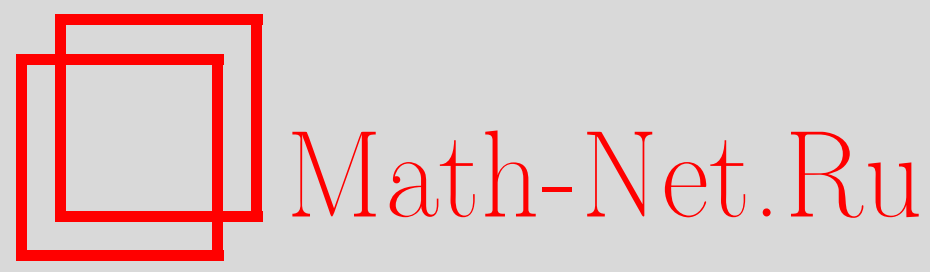

Г. В. Воскресенская, Мультипликативные произведения эта-функций Дедекинда и представления групп, Матем. заметки, 2003, том 73, выпуск 4, 511-526

DOI: https://doi.org/10.4213/mzm211

Использование Общероссийского математического портала Math-Net.Ru подразумевает, что вы прочитали и согласны с пользовательским соглашением http://www . mathnet.ru/rus/agreement

Параметры загрузки:

IP: 34.229 .45 .116

26 апреля 2023 г., $05: 27: 20$

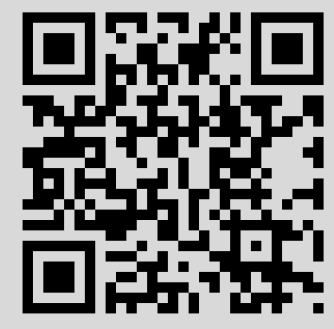




\section{МУЛЬТИПЛИКАТИВНЫЕ ПРОИЗВЕДЕНИЯ ЭТА-ФУНКЦИЙ ДЕДЕКИНДА И ПРЕДСТАВЛЕНИЯ ГРУПП}

\section{Г.В. Воскресенская}

В статье находятся все метациклические группы вида $\left\langle a, b: a^{m}=e, b^{s}=e, b^{-1} a b\right.$ $\left.=a^{r}\right\rangle$, где $m=10,14,15,20,21,22$, такие, что модулярные формы, ассоциированные со всеми элементами этих групп с помощью некоторого точного представления, являются мультипликативными $\eta$-произведениями. Также изучается соответствие между мультипликативными $\eta$-произведениями и элементами конечного порядка в $S L(5, C)$, задаваемое с помощью присоединенного представления.

Библиографоия: 14 названий.

1. Введение. Интересным актуальным аспектом теории модулярных форм является изучение их связей с представлениями конечных групп. В настоящей статье исследуется проблема нахождения таких конечных групा, что модулярные формы, ассоциированные со всеми элементами этих групा с помощью некоторого точного представления, принадлежат специальному классу модулярных форм, которыеназьваются мультипликативньми $\eta$-произведениями. Это открытая проблема: все такие групшы до сих пор не найдены. В этой статье продолжается исследование метациклических групा, начатое в статьях [1], [2]. Кроме того, рассматривается связь между мультипликативньми $\eta$-произведениями и элементами конечного порядка в $S L(5, C)$ с помошью присоединенного представления.

Эта-функиия,Дедекинда $\eta(z)$ определяется формулой

$$
\eta(z)=q^{1 / 24} \prod_{n=1}^{\infty}\left(1-q^{n}\right), \quad q=e^{2 \pi i z}
$$

$z$ лежит в верхней комплексной полуплоскости.

В этой статье рассматриваются модулярные формы, которые полностью описываются следующими условиями: это параболические формы целого веса с характерами, собственные относительно всех операторов Гекке и не имеющие нулей вне параболических вершин. Заранее мы не предполагаем, что эти функции являются модифицированными произведениями $\eta$-функций Дедекинда. Однако фактически это так, таких функций ровно 28; приведем их полньй список:

- формы веса 1:

$$
\begin{gathered}
\eta(23 z) \eta(z), \quad \eta(22 z) \eta(2 z), \quad \eta(21 z) \eta(3 z), \quad \eta(20 z) \eta(4 z) \\
\eta(18 z) \eta(6 z), \quad \eta(16 z) \eta(8 z), \quad \eta^{2}(12 z)
\end{gathered}
$$


- формы веса 2:

$$
\begin{aligned}
& \eta(15 z) \eta(5 z) \eta(3 z) \eta(z), \quad \eta(14 z) \eta(7 z) \eta(2 z) \eta(z), \quad \eta(12 z) \eta(6 z) \eta(4 z) \eta(2 z), \\
& \eta^{2}(11 z) \eta^{2}(z), \quad \eta^{2}(10 z) \eta^{2}(2 z), \quad \eta^{2}(9 z) \eta^{2}(3 z), \quad \eta^{2}(8 z) \eta^{2}(4 z), \quad \eta^{4}(6 z)
\end{aligned}
$$

- формы веса 3:

$$
\eta^{2}(8 z) \eta(4 z) \eta(2 z) \eta^{2}(z), \quad \eta^{3}(7 z) \eta^{3}(z), \quad \eta^{3}(6 z) \eta^{3}(2 z), \quad \eta^{6}(4 z)
$$

- формы веса 4:

$$
\eta^{4}(5 z) \eta^{4}(z), \quad \eta^{4}(4 z) \eta^{4}(2 z), \quad \eta^{2}(6 z) \eta^{2}(3 z) \eta^{2}(2 z) \eta^{2}(z), \quad \eta^{8}(3 z)
$$

- форма веса 5: $\eta^{4}(4 z) \eta^{2}(2 z) \eta^{4}(z)$;

- формы веса 6: $\eta^{6}(3 z) \eta^{6}(z), \eta^{12}(2 z)$;

- форма веса 8: $\eta^{8}(2 z) \eta^{8}(z)$;

- форма веса 12: $\eta^{24}(z)$.

$\mathrm{K}$ этому списку добавим еще 2 параболические формы полуцелого веса: $\eta(24 z), \eta^{3}(8 z)$.

Эти функции мы назовем мультипликативными $\eta$-произведениями, так как они имеют мультипликативные коэффициенты Фурье.

С различных точек зрения эти функции изучались в ряде недавних работ американских и японских математиков [3]-[9].

Сопоставление элементам конечных групп модулярных форм осуществляется по следующему правилу. Пусть $\Phi$ - представление конечной группы $G$ унимодулярньми матрицами в пространстве $V$, размерность которого делится на 24 . Тогда для любого элемента $g \in G$ характеристический многочлен оператора $\Phi(g)$ имеет вид

$$
P_{g}(x)=\prod_{k=1}^{s}\left(x^{a_{k}}-1\right)^{t_{k}}, \quad a_{k} \in \mathbb{N}, \quad t_{k} \in \mathbb{Z} .
$$

С каждьм элементом $g \in G$ можно связать функцию

$$
\eta_{g}(z)=\prod_{k=1}^{s} \eta^{t_{k}}\left(a_{k} z\right)
$$

Функция $\eta_{g}(z)$ является параболической формой определенного уровня $N(g)$ и веса

$$
k(g)=\frac{1}{2} \sum_{k=1}^{s} t_{k}
$$

с характером, равным характеру квадратичного поля

$$
\mathbf{Q} \sqrt{\prod_{k=1}^{s}\left(i a_{k}\right)^{t_{k}}}
$$


Метациклической группой назьвается конечная группа, имеющая циклический нормальный делитель, факторгруппа по которому также циклическая.

Генетический код метациклической группы $\left\langle a, b: a^{m}=e, b^{s}=e, b^{-1} a b=a^{r}\right\rangle$.

Будем называть представление групшы искомым или представлением допустимого типа, если с помощью этого представления с элементами групшы ассоциируются мультипликативные $\eta$-произведения. Целью работы является нахождение метациклических груп, имеющих такие точные представления. При разборе каждого случая будут подробно указаны искомые представления.

Допустимые групшы указьваются с точностью до изоморфизма.

Искомые групш могут содержать элементы порядков, не превосходящих 24 и не равных 13, 17, 19. Непосредственно проверяется, что если некоторому элементу групшы соответствует мультипликативное $\eta$-произведение, то всем его степеням также соответствуют параболические формы из указанного вьше списка. Используя этот факт, при исследовании групп достаточно рассматривать представления только для элементов, не лежащих в одной циклической группе. Единичному элементу групшы соответствует параболическая форма $\eta^{24}(z)$.

Так как искомое представление является точньп, по виду параболической формы можно понять порядок соответствующего ей элемента группы. Поэтому мы будем приводить только списки параболических форм, указьвая элементы только в том случае, когда элементам группы равных порядков соответствуют различные формы.

Если некоторая допустимая группа является подгруппой другой допустимой метациклической группы, то можно привести подробное рассмотрение только для большей группы. Результат для подгрупшы следует из него очевидным образом.

В этой статье полностью разобраны случаи, когда $m=10,14,15,20,21,22$, и циклические подгрупшы $\langle a\rangle$ и $\langle b\rangle$ не имеют нетривиального пересечения. Случаи $m=3,4,5,7$, 11,23 были разобраны в статье [1], случаи $m=9,18$ - в статье [2].

Основной результат статьи может быть сформулирован в виде следующей теоремы.

Теорема 1. Пусть $G$-метациклическая группа с генетическим кодом

$$
\left\langle a, b: a^{m}=e, \quad b^{s}=e, \quad b^{-1} a b=a^{r}\right\rangle, \quad m=10,14,15,20,21,22,
$$

такая, что с каждым әлементом этой группы с помощъю некоторого точного представления ассочиируется мультипликативное $\eta$-произведение. Тогда для $m, s, r$ возмохны ( $с$ точностью до изоморфизма) лишь указанные в таблице 1 значения.

ТАБЛИЦА 1

\begin{tabular}{|c|c|c|c|c|c|c|c|c|c|c|c|c|c|c|c|c|c|c|c|c|}
\hline$m$ & 10 & 10 & 10 & 10 & 14 & 14 & 14 & 15 & 15 & 15 & 20 & 20 & 20 & 21 & 21 & 21 & 21 & 22 & 22 & 22 \\
\hline$s$ & 4 & 8 & 2 & 4 & 6 & 3 & 2 & 4 & 2 & 2 & 2 & 4 & 2 & 6 & 3 & 2 & 2 & 5 & 10 & 2 \\
\hline$r$ & 3 & 3 & 9 & 9 & 3 & 9 & 13 & 2 & 4 & 14 & 9 & 17 & 19 & 2 & 4 & 8 & 20 & 3 & 7 & 21 \\
\hline
\end{tabular}

Диэдральные группы допустимых типов подробно рассматривались автором в предыдущей статье [10]. 
2. Метациклические группы вида $\left\langle a, b: a^{10}=e, b^{s}=e, b^{-1} a b=a^{r}\right\rangle$. Групша $D_{10}$ является допустимой, она была рассмотрена ранее.

2.1. Группа $\left\langle a, b: a^{10}=e, b^{4}=e, b^{-1} a b=a^{-1}\right\rangle$. Это группа порядка 40, она имеет 16 классов сопряженных элементов. Элементы $e, a^{5}, b^{2}, a^{5} b^{2}$ являются центральными.

Искомым представлением является прямая сумма всех неприводимых представлений, взятых с кратностью 1 . Элементам $a^{5}, b^{2}$ соответствует параболическая форма $\eta^{12}(2 z)$, элементу $a^{5} b^{2}$ соответствует $\eta^{8}(2 z) \eta^{8}(z)$, остальным элементам грушы соответствуют параболические формы

$$
\eta^{2}(10 z) \eta^{2}(2 z), \quad \eta^{4}(5 z) \eta^{4}(z), \quad \eta^{6}(4 z), \quad \eta^{24}(z)
$$

Другое искомое представление будет рассмотрено в следующем пункте как ограничение представления большей групш.

2.2. Групша $\left\langle a, b: a^{10}=e, b^{8}=e, b^{-1} a b=a^{3}\right\rangle$. Это группа порядка 80, она имеет 20 классов сопряженных элементов. Элементы $e, a^{5}, b^{4}, a^{5} b^{4}$ являются центральньми.

Искомым представлением является прямая сумма всех неприводимых представлений, взятых с кратностью 1, кроме тех одномерных, в которых элемент $b$ переходит в первообразные корни степени 8 из 1 . Элементам $a^{5}, a^{5} b^{4}$ соответствует параболическая форма $\eta^{12}(2 z)$, элементу $b^{4}$ соответствует $\eta^{8}(2 z) \eta^{8}(z)$, остальным элементам группы соответствуют параболические формы

$$
\eta^{2}(10 z) \eta^{2}(2 z), \quad \eta^{4}(5 z) \eta^{4}(z), \quad \eta^{2}(8 z) \eta^{2}(4 z), \quad \eta^{4}(4 z) \eta^{4}(2 z), \quad \eta^{24}(z)
$$

Она содержит в качестве подгруппы группу $\left\langle a, b: a^{10}=e, b^{4}=e, b^{-1} a b=a^{9}\right\rangle$, ограничение этого представления на нее отличается от представления, рассмотренного в 2.1.

2.3. Групша $\left\langle a, b: a^{10}=e, b^{4}=e, b^{-1} a b=a^{3}\right\rangle$. Это групша порядка 40 , она имеет 10 классов сопряженных элементов. Элементы $e, a^{5}$ являются центральными.

Искомым представлением является прямая сумма всех неприводимых представлений, взятых с кратностью 1, кроме тех одномерных, в которых элемент $b$ переходит в первообразные корни степени 4 из 1 . Элементам $a^{5}, a^{5} b^{2}$ соответствует параболическая форма $\eta^{12}(2 z)$, элементу $b^{2}$ соответствует $\eta^{8}(2 z) \eta^{8}(z)$, остальным элементам группы соответствуют параболические формы

$$
\eta^{2}(10 z) \eta^{2}(2 z), \quad \eta^{4}(5 z) \eta^{4}(z), \quad \eta^{4}(4 z) \eta^{4}(2 z), \quad \eta^{24}(z)
$$

Эта групша изоморфна группе $\left\langle a, b: a^{10}=e, b^{4}=e, b^{-1} a b=a^{7}\right\rangle$.

3. Метациклические группы вида $\left\langle a, b: a^{14}=e, b^{s}=e, b^{-1} a b=a^{r}\right\rangle$. Група $D_{14}$ является допустимой, она была рассмотрена ранее.

Группа $\left\langle a, b: a^{14}=e, b^{6}=e, b^{-1} a b=a^{3}\right\rangle$. Это группа порядка 84, она имеет 14 классов сопряженных элементов. Элемент $a^{7}$ является центральным.

Искомым представлением является прямая сумма

$$
T_{1} \oplus 2 T_{2} \oplus 2 T_{3} \oplus 2 T_{4} \oplus T_{5} \oplus T_{6}
$$


где

$$
\begin{gathered}
T_{1}(a)=\left(\begin{array}{cccccc}
\zeta_{14} & 0 & 0 & 0 & 0 & 0 \\
0 & \zeta_{14}^{3} & 0 & 0 & 0 & 0 \\
0 & 0 & \zeta_{14}^{9} & 0 & 0 & 0 \\
0 & 0 & 0 & \zeta_{14}^{13} & 0 & \\
0 & 0 & 0 & 0 & \zeta_{14}^{11} & 0 \\
0 & 0 & 0 & 0 & 0 & \zeta_{14}^{5}
\end{array}\right), \quad T_{1}(b)=T_{2}(b)=\left(\begin{array}{ccccccc}
0 & 0 & 0 & 0 & 0 & 1 \\
1 & 0 & 0 & 0 & 0 & 0 \\
0 & 1 & 0 & 0 & 0 & 0 \\
0 & 0 & 1 & 0 & 0 & 0 \\
0 & 0 & 0 & 1 & 0 & 0 \\
0 & 0 & 0 & 0 & 1 & 0
\end{array}\right) \\
T_{2}(a)=\left(\begin{array}{cccccc}
\zeta_{7} & 0 & 0 & 0 & 0 & 0 \\
0 & \zeta_{7}^{3} & 0 & 0 & 0 & 0 \\
0 & 0 & \zeta_{7}^{2} & 0 & 0 & 0 \\
0 & 0 & 0 & \zeta_{7}^{6} & 0 \\
0 & 0 & 0 & 0 & \zeta_{7}^{4} & 0 \\
0 & 0 & 0 & 0 & 0 & \zeta_{7}^{5}
\end{array}\right), \\
T_{5}(a)=-1, \quad T_{5}(b)=1, \quad T_{6}(a)=-1, \quad T_{6}(b)=-1 .
\end{gathered}
$$

Элементам $b^{3}, a^{7} b^{3}$ соответствует параболическая форма $\eta^{12}(2 z)$, элементу $a^{7}$ соответствует $\eta^{8}(2 z) \eta^{8}(z)$, остальным элементам группы соответствуют параболические формы

$$
\begin{gathered}
\eta^{3}(7 z) \eta^{3}(z), \quad \eta(21 z) \eta(3 z), \quad \eta(14 z) \eta(7 z) \eta(2 z) \eta(z), \quad \eta^{3}(6 z) \eta^{3}(2 z) \\
\eta^{6}(3 z) \eta^{6}(z), \quad \eta^{24}(z)
\end{gathered}
$$

Эта групша изоморфна группе $\left\langle a, b: a^{14}=e, b^{6}=e, b^{-1} a b=a^{5}\right\rangle$. Подгруппой в этой группе является группа $\left\langle a, b: a^{14}=e, b^{3}=e, b^{-1} a b=a^{9}\right\rangle$, которая изоморфна группе $\left\langle a, b: a^{14}=e, b^{3}=e, b^{-1} a b=a^{11}\right\rangle$.

4. Метациклические группы вида $\left\langle a, b: a^{15}=e, b^{s}=e, b^{-1} a b=a^{r}\right\rangle$. Група $D_{15}$ является допустимой, она была рассмотрена ранее.

Группа $H \cong\left\langle a, b: a^{15}=e, b^{4}=e, b^{-1} a b=a^{2}\right\rangle$. Это группа порядка 60, у нее 9 классов сопряженных элементов.

Пусть $T_{1}, T_{2}$ - неприводимые 4-мерные представления, переводящие элемент $a$ в элементы порядка 15,

$T_{3}$ - неприводимое 4-мерное представление, переводящее элемент $a$ в элемент порядка 5 ,

$$
\begin{gathered}
T_{4}(a)=\left(\begin{array}{cc}
\zeta_{3} & 0 \\
0 & \zeta_{3}^{2}
\end{array}\right), \quad T_{4}(b)=\left(\begin{array}{cc}
0 & i \\
i & 0
\end{array}\right), \\
T_{6}(a)=T_{7}(a)=T_{8}(a)=T_{9}(a)=1, \quad T_{6}(b)=-1 \\
T_{7}(b)=1, \quad T_{8}(a)=i, \quad T_{9}(b)=-i .
\end{gathered}
$$

Искомым представлением является прямая сумма

$$
T_{1} \oplus T_{2} \oplus 2 T_{3} \oplus T_{4} \oplus T_{5} \oplus T_{6} \oplus T_{7} \oplus T_{8} \oplus T_{9}
$$


или

$$
T_{1} \oplus T_{2} \oplus 2 T_{3} \oplus 2 T_{5} \oplus 2 T_{8} \oplus 2 T_{9} .
$$

Эта группа изоморфна группе $\left\langle a, b: a^{15}=e, b^{4}=e, b^{-1} a b=a^{8}\right\rangle$. В ней содержится подгрупша $\left\langle a, b: a^{15}=e, b^{2}=e, b^{-1} a b=a^{4}\right\rangle$.

5. Метациклические группы вида $\left\langle a, b: a^{20}=e, b^{s}=e, b^{-1} a b=a^{r}\right\rangle$. Группа $D_{20}$ является допустимой, она была рассмотрена ранее.

Группа $\left\langle a, b: a^{20}=e, b^{4}=e, b^{-1} a b=a^{17}\right\rangle$. Это группа порядка 80, у нее 20 классов сопряженных элементов.

Искомое представление является прямой суммой, в которую с кратностью 1 входят все неприводимые 4-мерные представления и все те неприводимые одномерные, в которых $b$ не переходит в первообразные корни степени 4 из 1.

Элементы, сопряженные с $a^{10}, a^{10} b^{2}$, соответствуют параболической форме $\eta^{12}(2 z)$; элементы, сопряженные с $b^{2}$, соответствуют $\eta^{8}(2 z) \eta^{8}(z)$; элементы, сопряженные с $b$, $b^{3}, a^{10} b, a^{5} b^{2}, a^{15} b^{2}, a^{10} b^{3}$ соответствуют $\eta^{4}(4 z) \eta^{4}(2 z)$; остальным элементам порядка 4 соответствует форма $\eta^{6}(4 z)$, остальным элементам группы соответствуют модулярные формы

$$
\eta(20 z) \eta(4 z), \quad \eta^{2}(10 z) \eta^{2}(2 z), \quad \eta^{4}(5 z) \eta^{4}(z), \quad \eta^{24}(z) .
$$

В этой группе содержится подгруппа $\left\langle a, b: a^{20}=e, b^{2}=e, b^{-1} a b=a^{9}\right\rangle$.

6. Метацик лические группы вида $\left\langle a, b: a^{21}=e, b^{s}=e, b^{-1} a b=a^{r}\right\rangle$. Групп $D_{21}$ является допустимой, она была рассмотрена ранее.

Групша $\left\langle a, b: a^{21}=e, b^{6}=e, b^{-1} a b=a^{5}\right\rangle$. Это группа порядка 126, у нее 12 классов сопряженных элементов.

Искомым представлением является прямая сумма

$$
2 T_{1} \oplus T_{4} \oplus T_{5} \oplus T_{6} \oplus T_{7} \oplus T_{8}
$$

или

$$
T_{2} \oplus T_{3} \oplus T_{4} \oplus T_{5} \oplus T_{6} \oplus T_{7} \oplus T_{8}
$$

где

$$
\begin{gathered}
T_{1}(a)=T_{2}(a)=T_{3}(a)=\left(\begin{array}{cc}
\zeta_{3} & 0 \\
0 & \zeta_{3}^{2}
\end{array}\right), \quad T_{1}(b)=\left(\begin{array}{cc}
0 & 1 \\
1 & 0
\end{array}\right), \quad T_{2}(b)=\left(\begin{array}{cc}
0 & \zeta_{3} \\
\zeta_{3} & 0
\end{array}\right), \\
T_{3}(b)=\left(\begin{array}{cc}
0 & \zeta_{3}^{2} \\
\zeta_{3}^{2} & 0
\end{array}\right), \quad T_{4}(a)=T_{5}(a)=1, \quad T_{4}(b)=1, \quad T_{5}(b)=-1
\end{gathered}
$$

$T_{6}, T_{7}, T_{8}$ - неприводимые 6-мерные представления.

В первом случае элементам, сопряженным с $b, a b^{2}, a b^{4}$, соответствует модулярная форма $\eta^{3}(6 z) \eta^{3}(2 z)$, элементам, сопряженным с $a^{7},-\eta^{8}(3 z)$, остальньм элементам порядка $3-\eta^{6}(3 z) \eta^{6}(z)$; во втором случае элементам, сопряженным с $a b^{2}, a b^{4}$ соответствует $\eta^{3}(6 z) \eta^{3}(2 z)$, элементам, сопряженным с $b$, соответствует $\eta^{4}(6 z)$, элементам, сопряженньм с $a^{7}, b^{2}-\eta^{8}(3 z)$, остальньм элементам порядка $3-\eta^{6}(3 z) \eta^{6}(z)$, элементам порядка 21 в обоих случаях соответствует $\eta(21 z) \eta(3 z)$, элементам порядка 7 - модулярная форма $\eta^{3}(7 z) \eta^{3}(z)$, элементам порядка 2 - модулярная форма $\eta^{12}(2 z)$, элементу $e$ соответствует $\eta^{24}(z)$. 
Эта групша изоморфна группам $\left\langle a, b: a^{21}=e, b^{6}=e, b^{-1} a b=a^{r}\right\rangle, r=2,11,17$. Она содержит в качестве подгрупшы групу $\left\langle a, b: a^{21}=e, b^{3}=e, b^{-1} a b=a^{4}\right\rangle$, которая, в свою очередь, изоморфна групе $\left\langle a, b: a^{21}=e, b^{3}=e, b^{-1} a b=a^{16}\right\rangle$, и подгруппу $\left\langle a, b: a^{21}=e, b^{2}=e, b^{-1} a b=a^{8}\right\rangle$.

7. Метациклические группы вида $\left\langle a, b: a^{22}=e, b^{s}=e, b^{-1} a b=a^{r}\right\rangle$. Группа $D_{22}$ является допустимой, она была рассмотрена ранее.

7.1. Групша $\left\langle a, b: a^{22}=e, b^{10}=e, b^{-1} a b=a^{7}\right\rangle$. Это группа порядка 220 , она имеет 22 класса сопряженных элементов. Элемент $a^{11}$ является центральным.

Искомым представлением является прямая сумма

$$
T_{1} \oplus T_{2} \oplus T_{3} \oplus T_{4} \oplus T_{5} \oplus T_{6},
$$

где

$$
\begin{aligned}
& T_{1}(a)=\left(\begin{array}{cccccccccc}
\zeta_{22}^{7} & 0 & 0 & 0 & 0 & 0 & 0 & 0 & 0 & 0 \\
0 & \zeta_{22}^{5} & 0 & 0 & 0 & 0 & 0 & 0 & 0 & 0 \\
0 & 0 & \zeta_{22}^{13} & 0 & 0 & 0 & 0 & 0 & 0 & 0 \\
0 & 0 & 0 & \zeta_{22}^{3} & 0 & 0 & 0 & 0 & 0 & 0 \\
0 & 0 & 0 & 0 & \zeta_{22}^{21} & 0 & 0 & 0 & 0 & 0 \\
0 & 0 & 0 & 0 & 0 & \zeta_{22}^{15} & 0 & 0 & 0 & 0 \\
0 & 0 & 0 & 0 & 0 & 0 & \zeta_{22}^{17} & 0 & 0 & 0 \\
0 & 0 & 0 & 0 & 0 & 0 & 0 & \zeta_{22}^{9} & 0 & 0 \\
0 & 0 & 0 & 0 & 0 & 0 & 0 & 0 & \zeta_{22}^{19} & 0 \\
0 & 0 & 0 & 0 & 0 & 0 & 0 & 0 & 0 & \zeta_{22}
\end{array}\right), \\
& T_{1}(b)=T_{2}(b)=\left(\begin{array}{cccccccccc}
0 & 0 & 0 & 0 & 0 & 0 & 0 & 0 & 0 & 1 \\
1 & 0 & 0 & 0 & 0 & 0 & 0 & 0 & 0 & 0 \\
0 & 1 & 0 & 0 & 0 & 0 & 0 & 0 & 0 & 0 \\
0 & 0 & 1 & 0 & 0 & 0 & 0 & 0 & 0 & 0 \\
0 & 0 & 0 & 1 & 0 & 0 & 0 & 0 & 0 & 0 \\
0 & 0 & 0 & 0 & 1 & 0 & 0 & 0 & 0 & 0 \\
0 & 0 & 0 & 0 & 0 & 1 & 0 & 0 & 0 & 0 \\
0 & 0 & 0 & 0 & 0 & 0 & 1 & 0 & 0 & 0 \\
0 & 0 & 0 & 0 & 0 & 0 & 0 & 1 & 0 & 0 \\
0 & 0 & 0 & 0 & 0 & 0 & 0 & 0 & 1 & 0
\end{array}\right), \\
& T_{2}(a)=\left(\begin{array}{cccccccccc}
\zeta_{11}^{7} & 0 & 0 & 0 & 0 & 0 & 0 & 0 & 0 & 0 \\
0 & \zeta_{11}^{5} & 0 & 0 & 0 & 0 & 0 & 0 & 0 & 0 \\
0 & 0 & \zeta_{11}^{13} & 0 & 0 & 0 & 0 & 0 & 0 & 0 \\
0 & 0 & 0 & \zeta_{11}^{3} & 0 & 0 & 0 & 0 & 0 & 0 \\
0 & 0 & 0 & 0 & \zeta_{11}^{21} & 0 & 0 & 0 & 0 & 0 \\
0 & 0 & 0 & 0 & 0 & \zeta_{11}^{15} & 0 & 0 & 0 & 0 \\
0 & 0 & 0 & 0 & 0 & 0 & \zeta_{11}^{17} & 0 & 0 & 0 \\
0 & 0 & 0 & 0 & 0 & 0 & 0 & \zeta_{11}^{9} & 0 & 0 \\
0 & 0 & 0 & 0 & 0 & 0 & 0 & 0 & \zeta_{11}^{19} & 0 \\
0 & 0 & 0 & 0 & 0 & 0 & 0 & 0 & 0 & \zeta_{11}
\end{array}\right), \\
& T_{3}(a)=1, \quad T_{3}(b)=1, \quad T_{4}(a)=1, \quad T_{4}(b)=-1, \\
& T_{5}(a)=-1, \quad T_{5}(b)=1, \quad T_{6}(a)=-1, \quad T_{6}(b)=-1 .
\end{aligned}
$$


Элементам групш соответствуют параболические формы

$$
\eta^{2}(10 z) \eta^{2}(2 z), \quad \eta(22 z) \eta(2 z), \quad \eta^{4}(5 z) \eta^{4}(z), \quad \eta^{2}(11 z) \eta^{2}(z), \quad \eta^{12}(2 z), \quad \eta^{24}(z)
$$

Эта группа изоморфна группам $\left\langle a, b: a^{22}=e, b^{10}=e, b^{-1} a b=a^{r}\right\rangle$, где $r=13,17,19$; она содержит подгруппу, изоморфную групе $\left\langle a, b: a^{22}=e, b^{5}=e, b^{-1} a b=a^{5}\right\rangle$, которая, в свою очередь, изоморфна группам $\left\langle a, b: a^{22}=e, b^{5}=e, b^{-1} a b=a^{r}\right\rangle$, где $r=3,9,15$.

\section{8. Метациклические группы, не имеющие представлений допустимого} типа. В этом пункте мы рассмотрим группы, элементы которых имеют допустимые порядки, но для самих групш не существует представлений искомого типа. Отсутствие искомого представления доказывается на основе анализа таблицы неприводимых представлений групшы, причем не обязательно рассматривать представления для всех элементов групшы. Если данная группа не имеет представлений допустимого типа, то это же верно и для любой группы, ее содержащей.

Все эти значения указаны в таблице 2 .

ТАБЛИЦА 2

\begin{tabular}{|c|c|c|c|c|c|c|c|c|c|c|c|c|c|c|c|c|c|c|c|c|}
\hline$m$ & 10 & 10 & 14 & 14 & 14 & 14 & 14 & 15 & 15 & 15 & 15 & 15 & 15 & 15 & 15 & 20 & 20 & 20 & 20 & 20 \\
\hline$s$ & 20 & 10 & 12 & 6 & 21 & 4 & 14 & 12 & 20 & 6 & 10 & 4 & 2 & 6 & 10 & 4 & 4 & 10 & 2 & 8 \\
\hline$r$ & 3 & 9 & 3 & 9 & 9 & 13 & 13 & 2 & 2 & 4 & 4 & 7 & 11 & 14 & 14 & 3 & 9 & 9 & 11 & 3 \\
\hline
\end{tabular}

\begin{tabular}{|c|c|c|c|c|c|c|c|c|c|c|c|c|c|c|c|}
\hline$m$ & 20 & 20 & 20 & 21 & 21 & 21 & 21 & 21 & 21 & 21 & 21 & 22 & 22 & 22 & 22 \\
\hline$s$ & 20 & 4 & 10 & 9 & 21 & 18 & 6 & 6 & 2 & 6 & 14 & 10 & 20 & 4 & 22 \\
\hline$r$ & 9 & 9 & 11 & 17 & 17 & 19 & 19 & 4 & 4 & 5 & 8 & 10 & 13 & 20 & 20 \\
\hline
\end{tabular}

Приведем доказательства для некоторых групा.

Группа $\left\langle a, b: a^{15}=e, b^{20}=e, b^{-1} a b=a^{2}\right\rangle$. Эта групша $G \cong Z_{5} \times H$, где $H$ - группа порядка 60 , описанная в начале п. 4. В искомом представлении должны содержаться 4 4-мерных представления, так как если $T$ - двумерное или одномерное представление, то среди собственных значений оператора $T(a)$ нет первообразных корней степени 15 из 1.

Пусть $T_{1}$ - представление степени 4, содержащееся в искомом. Если $T_{1}\left(b^{4}\right)$ не является тождественным оператором, то среди собственньх значений оператора $T_{1}\left(a^{5} b^{4}\right)$ либо встречаются повторяющиеся первообразные корни степени 15 из 1 , либо все 4 собственных значения совпадают с одним и тем же значением первообразного корня степени 5 из 1. Получим противоречие, так как элемент $a^{5} b^{4}$ должен соответствовать форме $\eta(15 z) \eta(5 z) \eta(3 z) \eta(z)$. Следовательно, во всех четырех 4-мерных представлениях, входящих в искомое, матрица оператора $T_{1}\left(b^{4}\right)$ является единичной, и среди собственных значений оператора $T_{1}(b)$ два первообразных корня степени 4 из 1 встречаются не менее четырех раз каждый, что противоречит тому, что $b$ должна соответствовать параболическая форма $\eta(20 z) \eta(4 z)$. Таким образом, нельзя построить представление для этой группы, которое удовлетворяло бы условию теоремы.

$\Gamma р у п а ~\left\langle a, b: a^{21}=e, b^{21}=e, b^{-1} a b=a^{4}\right\rangle$. Если $T$ - трехмерное неприводимое представление этой групп, то среди собственных значений оператора $T(g)$ не содержатся 
корни степени 3 из 1. Так как элементу 21-го порядка должна соответствовать модулярная форма $\eta(21 z) \eta(3 z)$, в искомом представлении содержатся ровно 6 одномерных, в двух из которых элемент $a$ переходит в 1 . Следовательно, элемент $a^{7} b^{3}$ может перейти в первообразный корень степени 21 не более чем в четырех из 6 рассматриваемых одномерных представлений. Значит, в искомое представление должно входить такое трехмерное представление $T_{1}$, что собственными значениями оператора $T_{1}\left(a^{7} b^{3}\right)$ являются первообразные корни степени 21 из 1 . Но так как $a^{7} b^{3}$ - элемент центральньй, эти собственные значения совпадают. Следовательно, элементу 21-го порядка $a^{7} b^{3}$ не может соответствовать модулярная форма $\eta(21 z) \eta(3 z)$, и допустимого представления рассматриваемой группы не существует.

Групша $\left\langle a, b: a^{22}=e, b^{4}=e, b^{-1} a b=a^{21}\right\rangle$. Элементам порядка 22 в искомом представлении соответствует параболическая форма $\eta(22 z) \eta(2 z)$. Среди неприводимых представлений, являюшихся прямыми слагаемьми искомого представления $T$, обязательно встречается такое представление $T_{1}$, что $T_{1}(a)$ имеет порядок $22, T_{1}\left(b^{2}\right)=-E$. В противном случае, если $T_{k}\left(b^{2}\right)=-E$, то $T_{k}(a)$ - элемент 11-го порядка, и если $T_{k}(a)$ - элемент порядка 22 , то $T_{k}\left(b^{2}\right)=E$. Тогда среди собственных значений оператора $T\left(a b^{2}\right)$ встретятся повторяюшиеся первообразные корни степени 22 из 1 , что недопустимо. Без ограничения общности можно считать, что

$$
T_{1}(a)=\left(\begin{array}{cc}
\zeta_{22} & 0 \\
0 & \zeta_{22}^{21}
\end{array}\right), \quad T_{1}\left(b^{2}\right)=\left(\begin{array}{cc}
-1 & 0 \\
0 & -1
\end{array}\right) .
$$

Пусть

$$
T_{2}(a)=\left(\begin{array}{cc}
\zeta_{22}^{12} & 0 \\
0 & \zeta_{22}^{10}
\end{array}\right) \text {. }
$$

Если $T_{2}\left(b^{2}\right)=-E$, то $T_{1}\left(a^{2} b^{2}\right)=T_{2}\left(a^{2} b^{2}\right) ;$ если $T_{2}\left(b^{2}\right)=E$, то $T_{1}\left(a b^{2}\right)=T_{2}\left(a b^{2}\right) . \mathrm{B}$ первом случае среди собственных значений оператора $T\left(a^{2} b^{2}\right)$, а во втором случае среди собственных значений оператора $T\left(a b^{2}\right)$, встретятся повторяющиеся первообразные корни степени 22 из 1 , что недопустимо.

Для других групп доказательство проводится аналогичными рассуждениями.

9. Мультипликативные $\eta$-произведения и присоединенное представление $S L(5, C)$. В этом пункте мы рассмотрим связи между изучаемыми модулярными формами и представлениями групп с иной точки зрения.

Tеорема 2. Пусть Ad - присоединенное представление группь $S L(5, C)$, и $g \in$ $S L(5, C), \operatorname{ord}(g) \neq 3,6,9,21$, таков, что характеристический многочлен оператора $\operatorname{Ad}(g)$ имеет вид

$$
P_{g}(x)=\prod_{k=1}^{s}\left(x^{a_{k}}-1\right)^{t_{k}}, \quad a_{k} \in \mathbb{N}, \quad t_{k} \in \mathbb{N}
$$

Тогда соответствующая параболическая форма $\eta_{g}(z)=\prod_{k=1}^{s} \eta^{t_{k}}\left(a_{k} z\right)$ является мультипликативным $\eta$-произведением веса $k(g)>1$, и все мультипликативнье $\eta$-произведения веса $k(g)>1$ мохсно получить этим путем.

Eсли $\operatorname{ord}(g)=3,6,9,21$, то этим путем можнно получить все мультипликативные $\eta$-произведения веса $k(g)>1$. Кроме этого, в этом соответствии 
возникают пять модулярных форм, которые не являются мультипликативными п-произведениями:

$$
\eta^{4}(3 z) \eta^{12}(z), \quad \eta^{7}(3 z) \eta^{3}(z), \quad \eta^{2}(6 z) \eta^{6}(2 z), \quad \eta^{2}(9 z) \eta(3 z) \eta^{3}(z), \quad \eta(21 z) \eta^{3}(z)
$$

ДокАЗАтЕльство. Пусть $T: S L(5, \mathbb{C}) \rightarrow G L(V)$ - естественное представление $S L(5, C)$ в 5-мерном векторном пространстве $V ; T^{*}: S L(5, \mathbb{C}) \rightarrow G L\left(V^{*}\right)$ - сопряженное представление.

Рассмотрим представление $T \otimes T^{*}: S L(5, \mathbb{C}) \rightarrow G L\left(V \otimes V^{*}\right)$. Это представление раскладьвается в прямую сумму $T_{1} \oplus T_{2}$, где $T_{1}$ - присоединенное представление Ad группы $S L(5, \mathbb{C})$ в 24 -мерном пространстве, $T_{2}$ - одномерное тождественное представление.

Пусть $\lambda_{1}, \lambda_{2}, \lambda_{3}, \lambda_{4}, \lambda_{5}$ - собственные значения оператора $T(g)$. Элементы $\lambda_{k} / \lambda_{m}$, $1 \leqslant l, m \leqslant 5$, являются собственными значениями оператора $T \otimes T^{*}(g)$. Исключая одно собственное значение, равное 1 , получим множество собственных значений оператора $\operatorname{Ad}(g)$.

Среди собственных значений оператора $\operatorname{Ad}(g)$ имеется не менее четырех единиц; следовательно, вес ассоциированной с $g$ модулярной формы $\eta_{g}(z)$ больше 1.

Количество собственных значений оператора $\operatorname{Ad}(g)$, равных 1 , определяется тем, сколько равных собственных значений было у оператора $T(g)$. Это соответствие описывается приведенной ниже таблицей 3 . Одинаковыми буквами обозначены одинаковые значения, разными буквами - разные.

ТАБЛИЦА 3

\begin{tabular}{|c|c|}
\hline Собственные значения $T(g)$ & $\begin{array}{c}\text { Количество единиц } \\
\text { среди собственных значений } \\
\text { оператора } \operatorname{Ad}(g)\end{array}$ \\
\hline$(a, b, c, d, e)$ & 4 \\
\hline$(a, a, b, c, d)$ & 6 \\
\hline$(a, a, b, b, c)$ & 8 \\
\hline$(a, a, a, b, c)$ & 10 \\
\hline$(a, a, a, b, b)$ & 12 \\
\hline$(a, a, a, a, b)$ & 16 \\
\hline$(a, a, a, a, a)$ & 24 \\
\hline
\end{tabular}

Исследуем теперь задачу отдельно для каждого порядка от 1 до 24. Единичному элементу соответствует $\eta^{24}(z)$. Через $P_{g}(x)$ обозначим характеристический многочлен оператора $\operatorname{Ad}(g)$; через $\zeta_{m}$ - первообразньй корень степени $m$ из 1 ; через $\Phi_{m}-m$-й круговой многочлен. Везде далее через $s$ будем обозначать количество единиц среди собственных значений оператора $\mathrm{Ad}(g)$. В ходе доказательства будут явно указаны наборы собственных значений для оператора $T(g)$.

Случай $\operatorname{ord}(g)=2$. Имеем

$$
P_{g}(x)=\left(x^{2}-1\right)^{k}(x-1)^{m}, \quad 2 k+m=24, \quad s=k+m, \quad 0<k, \quad 0 \leqslant m .
$$


Из приведенных в таблище значений этим условиям удовлетворяют лишш $k=12, m=0$; $k=8, m=8$. Модулярной форме $\eta^{12}(2 z)$ соответствует набор собственных значений оператора $T(g)(1,1,1,-1,-1)$, а форме $\eta^{8}(2 z) \eta^{8}(z)$ соответствует $(1,-1,-1,-1,-1)$.

Случай $\operatorname{ord}(g)=3$. Имеем

$$
P_{g}(x)=\left(x^{3}-1\right)^{k}(x-1)^{m}, \quad 3 k+m=24, \quad s=k+m, \quad 0<k, \quad 0 \leqslant m .
$$

Из приведенных в таблице значений этим условиям удовлетворяют $k=8, m=0$; $k=7, m=3 ; k=6, m=6 ; k=4, m=12$. Модулярной форме $f_{1}=\eta^{8}(3 z)$ соответствует набор собственных значений $\left(\zeta_{3}, \zeta_{3}, \zeta_{3}^{2}, \zeta_{3}^{2}, 1\right)$ оператора $T(g)$, а форме $f_{2}=$ $\eta^{6}(3 z) \eta^{6}(z)$ соответствует $\left(\zeta_{3}, \zeta_{3}, \zeta_{3}, 1,1\right)$. Эти две функции являются мультипликативными $\eta$-произведениями. Функции $f_{3}=\eta^{7}(3 z) \eta^{3}(z)$ и $f_{4}=\eta^{4}(3 z) \eta^{12}(z)$ не являются мультипликативными $\eta$-произведениями. $Ф$ ункции $f_{3}$ соответствует набор собственных значений $\left(\zeta_{3}, \zeta_{3}^{2}, 1,1,1\right), f_{4}$ соответствует набор $\left(\zeta_{3}, \zeta_{3}, \zeta_{3}, \zeta_{3}, \zeta_{3}^{2}\right)$. Отметим любопытные соотношения

$$
f_{3}^{2}=f_{1} f_{2}, \quad f_{2}^{2}=f_{1} f_{4}
$$

Случай $\operatorname{ord}(g)=4$. Имеем

$$
P_{g}(x)=\left(x^{4}-1\right)^{k}\left(x^{2}-1\right)^{m}(x-1)^{l}, \quad 4 k+2 m+l=24, \quad s=k+m+l, \quad 2 \mid(k+m) .
$$

Характеристический многочлен можно представить в виде произведения круговых многочленов; $P_{g}=\Phi_{4}^{k} \Phi_{2}^{k+m} \Phi_{1}^{k+m+l}$.

Рассмотрим характеристический многочлен оператора $\operatorname{Ad}\left(g^{2}\right) P_{g^{2}}=\Phi_{2}^{2 k} \Phi_{1}^{2 k+2 m+l}$. Имеются возможности: $P_{g^{2}}(x)=\left(x^{2}-1\right)^{12}$ или $P_{g^{2}}(x)=\left(x^{2}-1\right)^{8}(x-1)^{8}$.

В первом случае $2 k=12,2 k+2 m+l=12$, во втором случае $2 k=8,2 k+2 m+l=16$.

Этим условиям удовлетворяют $k=6, m=0, l=0 ; k=4, m=4, l=0 ; k=4$, $m=2, l=4 ; k=4, m=0, l=8$.

В последнем случае набор собственных значений должен иметь вид $(a, a, a, b, b)$. Но в этом случае среди отношений $\lambda_{k} / \lambda_{m}$ имеется не более двух различных значений, не равных 1 , а среди собственных значений оператора $\operatorname{Ad}(g)$ должны содержаться числа $i,-i$, -1. Получили противоречие. Три других варианта соответствуют мультипликативным $\eta$-произведениям. Модулярной форме $\eta^{6}(4 z)$ соответствует набор собственных значений оператора $T(g)(i,-i,-1,-1,1)$, форме $\eta^{4}(4 z) \eta^{4}(2 z)$ соответствует $(i,-i, 1,1,1)$, а форме $\eta^{4}(4 z) \eta^{2}(2 z) \eta^{4}(z)$ соответствует $(i,-i, i,-i, 1)$.

Случай $\operatorname{ord}(g)=5$. Имеем

$$
P_{g}(x)=\left(x^{5}-1\right)^{k}(x-1)^{m}, \quad 5 k+m=24, \quad s=k+m, \quad 0<k \leqslant 4, \quad 0 \leqslant m .
$$

Получим $s=24-4 k$; следовательно, $4 \mid s$. Так как среди отношений $\lambda_{k} / \lambda_{m}$ должно быть не менее 4 различных неединичных значений, то $s \leqslant 10$. С учетом этих условий возможен единственный вариант $k=4, m=4$. Модулярной форме $\eta^{4}(5 z) \eta^{4}(z)$ соответствует набор собственных значений оператора $T(g)\left(\zeta_{5}, \zeta_{5}, \zeta_{5}^{2}, \zeta_{5}^{2}, \zeta_{5}^{4}\right)$.

Случай $\operatorname{ord}(g)=6$. Имеем

$$
\begin{gathered}
P_{g}(x)=\left(x^{6}-1\right)^{k}\left(x^{3}-1\right)^{m}\left(x^{2}-1\right)^{l}(x-1)^{n}, \\
6 k+3 m+2 l+n=24, \quad s=k+m+l+n, \quad k+l-\text { четное. }
\end{gathered}
$$


Так как среди отношений $\lambda_{k} / \lambda_{m}$ должно быть не менее 5 различных неединичных значений, то $s \leqslant 10$. С другой стороны, $P_{g}=\Phi_{6}^{k} \Phi_{3}^{k+m} \Phi_{2}^{k+l} \Phi_{1}^{k+m+l+n}$. Рассмотрим характеристический многочлен $P_{g^{3}}=\Phi_{2}^{3 k+l} \Phi_{1}^{3 k+3 m+l+n}$ оператора $\operatorname{Ad}\left(g^{3}\right)$.

Имеются 2 возможности: $P_{g^{2}}(x)=\left(x^{2}-1\right)^{12}$ или $P_{g^{2}}(x)=\left(x^{2}-1\right)^{8}(x-1)^{8}$.

В первом случае $3 k+l=12,3 k+3 m+l+n=12$, во втором случае $3 k+l=8$, $3 k+3 m+l+n=16$. Далее рассмотрим характеристический многочлен оператоpa $\operatorname{Ad}\left(g^{2}\right) P_{g^{2}}=\Phi_{3}^{2 k+m} \Phi_{1}^{2 k+m+2 l+n}$. Имеются 4 возможности: $2 k+m=8,2 k+m+$ $2 l+n=8 ; 2 k+m=6,2 k+m+2 l+n=12 ; 2 k+m=4,2 k+m+2 l+n=16 ; 2 k+m=7$, $2 k+m+2 l+n=10$. Так как при $k=0 l=8$ или $l=12$, a $4 \leqslant m$, то, очевидно, этот случай исключается. Следовательно, и при $\operatorname{ord}(g)=12,18,24$ в разложение $P_{g}(x)$ должен входить множитель $\left(x^{\mathrm{ord}}(g)-1\right)$. Учитывая все условия, при $0<k$ получим 4 варианта. Модулярной форме $\eta^{4}(6 z)$ соответствует набор собственных значений оператора $T(g)\left(\zeta_{6}, \zeta_{6}^{2}, \zeta_{6}^{4}, \zeta_{6}^{5}, 1\right)$, форме $\eta^{3}(6 z) \eta^{3}(2 z)$ соответствует $\left(\zeta_{6}, \zeta_{6}^{3}, \zeta_{6}^{4}, \zeta_{6}^{4}, 1\right)$, а форме $\eta^{2}(6 z) \eta^{2}(3 z) \eta^{2}(2 z) \eta^{2}(z)$ соответствует $\left(\zeta_{6}, \zeta_{6}, \zeta_{6}^{3}, \zeta_{6}^{3}, \zeta_{6}^{4}\right)$. Эти три модулярные формы являются мультипликативными $\eta$-произведениями. Здесь возникает еще одна функция, которая уже не является мультипликативным $\eta$-произведением. Это модулярная форма $\eta^{2}(6 z) \eta^{6}(2 z)$. Ей соответствует набор собственных значений $\left(\zeta_{6}^{2}, \zeta_{6}^{2}, \zeta_{6}^{4}, \zeta_{6}^{5}, \zeta_{6}^{5}\right)$.

Случай $\operatorname{ord}(g)=7$. Имеем

$$
P_{g}(x)=\left(x^{7}-1\right)^{k}(x-1)^{m}, \quad 7 k+m=24, \quad s=k+m, \quad 0<k \leqslant 3, \quad 0 \leqslant m .
$$

Получим $s=24-6 k$; следовательно, $6 \mid s$. Так как среди отношений $\lambda_{k} / \lambda_{m}$ должно быть не менее 6 различных неединичных значений, то $s \leqslant 10$. С учетом этих условий возможен единственный вариант $k=3, m=3$. Модулярной форме $\eta^{3}(7 z) \eta^{3}(z)$ соответствует набор собственных значений оператора $T(g)\left(\zeta_{7}, \zeta_{7}^{2}, \zeta_{7}^{4}, 1,1\right)$.

Случай $\operatorname{ord}(g)=8$. Имеем

$$
\begin{gathered}
P_{g}(x)=\left(x^{8}-1\right)^{k}\left(x^{4}-1\right)^{m}\left(x^{2}-1\right)^{l}(x-1)^{n}, \\
8 k+4 m+2 l+n=24, \quad s=k+m+l+n, \quad k+m+l-\text { четное; }
\end{gathered}
$$

$P_{g}=\Phi_{8}^{k} \Phi_{4}^{k+m} \Phi_{2}^{k+m+l} \Phi_{1}^{k+m+l+n}$. Рассмотрим характеристический многочлен оператора $\operatorname{Ad}\left(g^{4}\right) P_{g^{4}}=\Phi_{2}^{4 k} \Phi_{1}^{4 k+4 m+2 l+n}$.

Имеются 2 возможности: $P_{g^{4}}(x)=\left(x^{2}-1\right)^{12}$ или $P_{g^{4}}(x)=\left(x^{2}-1\right)^{8}(x-1)^{8}$.

В первом случае $4 k=12,4 k+4 m+2 l+n=12$, во втором случае $4 k=8,4 k+4 m+$ $2 l+n=16$.

Этим условиям удовлетворяют $k=3, m=0, l=0, n=0 ; k=2, m=2, l=0, n=0$; $k=2, m=1, l=1, n=2$.

Так как среди собственных значений оператора $\operatorname{Ad}(g)$ должно быть не менее 4 единиц, то вариант $P_{g}(x)=\left(x^{8}-1\right)^{3}$ невозможен. Два других варианта соответствуют мультипликативньм $\eta$-произведениям. Модулярной форме $\eta^{2}(8 z) \eta^{2}(4 z)$ соответствует набор собственных значений оператора $T(g)\left(\zeta_{8}, \zeta_{8}^{3}, \zeta_{8}^{5}, \zeta_{8}^{7}, 1\right)$, а форме $\eta^{2}(8 z) \eta(4 z) \eta(2 z) \eta^{2}(z)$ соответствует $\left(\zeta_{8}^{3}, \zeta_{8}^{3}, \zeta_{8}^{5}, \zeta_{8}^{6}, \zeta_{8}^{7}\right)$.

Случай $\operatorname{ord}(g)=9$. Имеем

$$
P_{g}(x)=\left(x^{9}-1\right)^{k}\left(x^{3}-1\right)^{m}(x-1)^{l}, \quad 9 k+3 m+l=24, \quad s=k+m+l, \quad 1 \leqslant k \leqslant 2 .
$$


С другой стороны, $P_{g}=\Phi_{9}^{k} \Phi_{3}^{k+m} \Phi_{1}^{k+m+l}$. Рассмотрим характеристический многочлен оператора $\operatorname{Ad}\left(g^{3}\right) P_{g^{3}}=\Phi_{3}^{3 k} \Phi_{1}^{3 k+3 m+l}$. Единственный возможньй вариант $3 k=6$, $3 k+3 m+l=12$. Так как среди отношений $\lambda_{k} / \lambda_{m}$ должно быть не менее 8 различных неединичных значений, то $s=4$ или $s=6$. Пусть $s=6$. Тогда $k=2,3 m+l=6, m+l=4$. Получим $k=2, m=1, l=3$. То есть $P_{g}(x)=\left(x^{9}-1\right)^{2}\left(x^{3}-1\right)(x-1)^{3}$. Искомьй набор собственных значений имеет вид $\left(\zeta_{9}, \zeta_{9}^{3}, \zeta_{9}^{3}, \zeta_{9}^{4}, \zeta_{9}^{7}\right)$. Модулярная форма $\left.\eta^{2}(9 z) \eta^{(} 3 z\right) \eta^{3}(z)$ не является мультипликативным $\eta$-произведением. Пусть $s=4$. Тогда $k=2,3 m+l=6$, $m+l=2$. Получим $k=2, m=2, l=0$. То есть $P_{g}(x)=\left(x^{9}-1\right)^{2}\left(x^{3}-1\right)^{2}$. Искомый набор собственных значений имеет вид $\left(\zeta_{9}^{2}, \zeta_{9}^{3}, \zeta_{9}^{5}, \zeta_{9}^{8}, 1\right)$. Модулярная форма $\eta^{2}(9 z) \eta^{2}(3 z)$ является мультипликативньм $\eta$-произведением.

Случай $\operatorname{ord}(g)=10$. Имеем

$$
\begin{gathered}
P_{g}(x)=\left(x^{10}-1\right)^{k}\left(x^{5}-1\right)^{m}\left(x^{2}-1\right)^{l}(x-1)^{n}, \\
10 k+5 m+2 l+n=24, \quad s=k+m+l+n, \quad 4 \leqslant s \leqslant 6, \quad 0 \leqslant k \leqslant 2, \quad k+l-\text { четное. }
\end{gathered}
$$

С другой стороны, $P_{g}=\Phi_{10}^{k} \Phi_{5}^{k+m} \Phi_{2}^{k+l} \Phi_{1}^{k+m+l+n}$. Рассмотрим характеристический многочлен оператора $\operatorname{Ad}\left(g^{5}\right) P_{g^{5}}=\Phi_{2}^{5 k+l} \Phi_{1}^{5 k+5 m+l+n}$.

Так как при $k=0 m=4$, a $l=8$ или $l=12$, то, очевидно, этот случай исключается. Следовательно, и при $\operatorname{ord}(g)=20$ в разложение $P_{g}(x)$ должен входить множитель $\left(x^{20}-1\right)$. При $0<k$ имеются 2 возможности: $P_{g^{5}}(x)=\left(x^{2}-1\right)^{12}$ или $P_{g^{5}}(x)=\left(x^{2}-1\right)^{8}(x-1)^{8}$.

В первом случае $5 k+l=12,5 k+5 m+l+n=12$, во втором случае $5 k+l=8$, $5 k+5 m+l+n=16$. Этим условиям удовлетворяет единственньй набор $k=2, m=0$, $l=2, n=0$. Модулярной форме $\eta^{2}(10 z) \eta^{2}(2 z)$ соответствует набор собственных значений $\left(\zeta_{10}^{3}, \zeta_{10}^{4}, \zeta_{10}^{6}, \zeta_{10}^{8}, \zeta_{10}^{9}\right)$ оператора $T(g)$.

Случай $\operatorname{ord}(g)=11$. Имеем

$$
P_{g}(x)=\left(x^{11}-1\right)^{k}(x-1)^{m}, \quad 11 k+m=24, \quad s=k+m, \quad 0<k \leqslant 2, \quad 0 \leqslant m .
$$

Получим $s=24-10 k$; следовательно, $2 \mid s$. Так как среди отношений $\lambda_{k} / \lambda_{m}$ должно быть не менее 10 различных неединичных значений, то $s \leqslant 6$. С учетом этих условий возможен единственный вариант $k=2, m=2$. Модулярной форме $\eta^{2}(11 z) \eta^{2}(z)$ соответствует набор собственных значений $\left(\zeta_{11}, \zeta_{11}^{3}, \zeta_{11}^{4}, \zeta_{11}^{5}, \zeta_{11}^{9}\right)$ оператора $T(g)$.

Случай $\operatorname{ord}(g)=12$. Имеем

$$
P_{g}(x)=\left(x^{12}-1\right)^{k}\left(x^{6}-1\right)^{m}\left(x^{4}-1\right)^{l}\left(x^{3}-1\right)^{n}\left(x^{2}-1\right)^{u}(x-1)^{v},
$$

$10 k+5 m+2 l+n=24, \quad s=k+m+l+n, \quad 4 \leqslant s \leqslant 6, \quad 0<k \leqslant 2, \quad k+l$-четное;

$$
\begin{gathered}
P_{g}=\Phi_{12}^{k} \Phi_{6}^{k+m} \Phi_{4}^{k+l} \Phi_{3}^{k+m+n} \Phi_{2}^{k+m+l+u} \Phi_{1}^{k+m+l+n+u+v} ; \\
P_{g^{6}}=\Phi_{2}^{6 k+2 l} \Phi_{1}^{6 k+6 m+2 l+3 n+2 u+v} .
\end{gathered}
$$

Если $P_{g^{6}}(x)=\left(x^{2}-1\right)^{12}$, то $6 k+2 l=12$. При $k=2 s=2$. Противоречие. Оставшийся вариант $P_{g}(x)=\left(x^{12}-1\right)\left(x^{3}-1\right)^{4}$. Тогда $P_{g^{2}}(x)=\left(x^{6}-1\right)^{2}\left(x^{2}-1\right)^{6} ; P_{g^{3}}=\left(x^{4}-1\right)^{6}$. Элементу $g^{2}$ соответствует набор собственных значений $\left(\zeta_{6}^{2}, \zeta_{6}^{2}, \zeta_{6}^{4}, \zeta_{6}^{5}, \zeta_{6}^{5}\right)$. Следовательно, среди собственных значений оператора $T(g)$ нет значений 1 и -1 . Элементу $g^{3}$ 
соответствует набор собственных значений $\left(\zeta_{4}, \zeta_{4}^{3},-1,-1,1\right)$. Следовательно, среди собственных значений оператора $T(g)$ встретятся 2 одинаковых первообразных корня степени 6 из 1. Это противоречит тому, что среди собственных значений оператора $\operatorname{Ad}(g)$ имеется ровно 4 единицы, и все числа в наборе $(a, b, c, d, e)$ должны быть различны.

Если $P_{g^{6}}(x)=\left(x^{2}-1\right)^{8}(x-1)^{8}$, то $k=1, l=1,6 m+3 n+2 u+v=8$. В этом случае $P_{g^{2}}(x)=\left(x^{6}-1\right)^{2}\left(x^{3}-1\right)^{2}\left(x^{2}-1\right)^{2}(x-1)^{2}$.

С другой стороны, $P_{g^{2}}=\Phi_{6}^{2} \Phi_{3}^{4+2 m+n} \Phi_{2}^{4} \Phi_{1}^{4+2 m+n+2 u+v}$. Получим $2 m+n=2$, $2 u+v=2$, a $P_{g^{3}}=\Phi_{4}^{4} \Phi_{2}^{4+3 m+u} \Phi_{1}^{4+3 m+3 n+u+v}$.

Если $P_{g^{3}}(x)=\left(x^{4}-1\right)^{4}\left(x^{2}-1\right)^{4}$, то $3 m+u=4,3 n+v=0$. Следовательно, $m=1$, $n=0, u=1, v=0$. Модулярной форме $\eta(12 z) \eta(6 z) \eta(4 z) \eta(2 z)$ соответствует набор собственных значений $\left(\zeta_{12}, \zeta_{12}^{3}, \zeta_{12}^{4}, \zeta_{12}^{7}, \zeta_{12}^{9}\right)$. Если $P_{g^{3}}(x)=\left(x^{4}-1\right)^{4}\left(x^{2}-1\right)^{2}(x-1)^{4}$, то $3 m+u=2,3 n+v=4$. Система уравнений $3 m+u=2,3 n+v=4,2 m+n=2$, $2 u+v=2$ не имеет решений в неотрицательных цельх числах.

Случай $\operatorname{ord}(g)=14$. Имеем

$$
P_{g}(x)=\left(x^{14}-1\right)^{k}\left(x^{7}-1\right)^{m}\left(x^{2}-1\right)^{l}(x-1)^{n}, \quad 14 k+7 m+2 l+n=24,
$$

$s=k+m+l+n-$ количество единиц среди собственных значений оператора $\operatorname{Ad}(g)$, $k+l$ - число четное. Так как при $k=0 m=3$, a $l=8$ или $l=12$, то, очевидно, этот случай исключается. Пусть $k=1$. Так как среди отношений $\lambda_{k} / \lambda_{m}$ должно быть не менее 13 различных неединичных значений, то $s=4$. С учетом этих условий возможен единственньй вариант $k=1, m=1, l=1, n=1$. Модулярной форме $\eta(14 z) \eta(7 z) \eta(2 z) \eta(z)$ соответствует набор собственных значений оператора $T(g)\left(\zeta_{14}^{3}, \zeta_{14}^{5}, \zeta_{14}^{7}, \zeta_{14}^{13}, 1\right)$.

Случай $\operatorname{ord}(g)=15$. Имеем

$P_{g}(x)=\left(x^{15}-1\right)^{k}\left(x^{5}-1\right)^{m}\left(x^{3}-1\right)^{l}(x-1)^{n}, \quad 15 k+5 m+3 l+n=24, \quad s=k+m+l+n$.

Так как при $k=0 m=4$, а $6 \leqslant l$, то, очевидно, этот случай исключается. Пусть $k=1$. Так как среди отношений $\lambda_{k} / \lambda_{m}$ должно быть в этом случае не менее 14 различных неединичных значений, то $s=4$. С учетом этих условий возможен единственный вариант $k=1, m=1, l=1, n=1$. Модулярной форме $\eta(15 z) \eta(5 z) \eta(3 z) \eta(z)$ соответствует набор собственных значений оператора $T(g)\left(\zeta_{15}, \zeta_{15}^{3}, \zeta_{15}^{4}, \zeta_{15}^{9}, \zeta_{15}^{13}\right)$.

Случай $\operatorname{ord}(g)=21$. Пусть $k=0$. Тогда $P_{g}(x)=\left(x^{7}-1\right)^{n}\left(x^{3}-1\right)^{m}(x-1)^{l}, n=3$, $4 \leqslant m$. Очевидно, этот случай исключается.

Пусть $k=1$. Тогда $P_{g}(x)=\left(x^{21}-1\right)^{k}\left(x^{3}-1\right)^{m}(x-1)^{l}, 21 k+3 m+l=24$, $s=k+m+l$. Так как среди отношений $\lambda_{k} / \lambda_{m}$ должно быть не менее 20 различных неединичных значений, то $s=4$. Возможен единственньй вариант $k=1, m=0$, $l=3$. Модулярной форме $\eta(21 z) \eta^{3}(z)$ соответствует набор собственных значений оператора $T(g)\left(\zeta_{21}^{3}, \zeta_{21}^{6}, \zeta_{21}^{7}, \zeta_{21}^{12}, \zeta_{21}^{14}\right)$. Эта модулярная форма не является мультипликативным $\eta$-произведением.

Случаи $\operatorname{ord}(g)=13,16,17,18,19,20,22,23,24$. В этих случаях в разложение $P_{g}(x)$ должен входить множитель $\left(x^{\operatorname{ord}(g)}-1\right)$. В случае $\operatorname{ord}(g)=22$, если это не так, то в разложение $P_{g}(x)$ должен входить множитель $\left(x^{2}-1\right)$ в степени 8 или 12 , что невозможно. Для остальных порядков это либо очевидно, либо обсуждалось ранее. Так как среди собственных значений оператора $\operatorname{Ad}(g)$ должно быть не менее 4 единищ, то исключается вариант $\operatorname{ord}(g)=22,23,24$. 
Пусть $\operatorname{ord}(g)=16$. Тогда $P_{g}(x)=\left(x^{16}-1\right)^{k}\left(x^{8}-1\right)^{m}\left(x^{4}-1\right)^{l}\left(x^{2}-1\right)^{n}(x-1)^{t}, k=1$, $8 m+4 l+2 n+t=8, s=m+l+n+t+1-$ количество единиц среди собственных значений оператора $\operatorname{Ad}(g), 4 \leqslant s \leqslant 6$; с другой стороны, $P_{g}=\Phi_{16} \Phi_{8}^{1+m} \Phi_{4}^{1+m+l} \Phi_{2}^{1+m+l+n} \times$ $\Phi_{1}^{1+m+l+n+t}$. Условие $8 m+4 l+2 n+t=8$ показьвает, что возможны два случая: $m=1$ или $m=0$. В первом случае $s=2$. Рассмотрим $P_{g^{2}}(x)=\Phi_{8}^{2} \Phi_{4}^{2+2 m} \Phi_{2}^{2+2 m+2 l} \times$ $\Phi_{1}^{2+2 m+2 l+2 n+t}$. Допустимые возможности: $P_{g^{2}}=\left(x^{8}-1\right)^{2}\left(x^{4}-1\right)^{2}$ или $P_{g^{2}}(x)=$ $\left(x^{8}-1\right)^{2}\left(x^{4}-1\right)\left(x^{2}-1\right)(x-1)^{2}$. При $m=0$ оба эти варианта невозможны.

Пусть $\operatorname{ord}(g)=18$. Тогда $P_{g}(x)=\left(x^{18}-1\right)^{k}\left(x^{6}-1\right)^{m}\left(x^{3}-1\right)^{l}\left(x^{2}-1\right)^{n}(x-1)^{t}$, $k=1,6 m+3 l+2 n+t=6, s=m+l+n+t+1-$ количество единиц среди собственных значений оператора $\operatorname{Ad}(g), 4 \leqslant s$.

Условие $6 m+3 l+2 n+t=6$ показывает, что возможны два случая: $m=1$ или $m=0$. В первом случае $s=2$. Рассмотрим $P_{g^{2}}=\Phi_{9}^{2} \Phi_{3}^{2+2 m+l} \Phi_{1}^{2+2 m+l+2 n+t}$. Допустимы две возможности: $P_{g^{2}}(x)=\left(x^{9}-1\right)^{2}\left(x^{3}-1\right)^{2}$ или $P_{g^{2}}(x)=\left(x^{9}-1\right)^{2}\left(x^{3}-1\right)(x-1)^{3}$. В первом случае получим $2 m+l=2$. При $m=0 s=3$, а при $m=1 s=2$. Во втором случае получим $2 m+l=1$. Тогда $k=1, l=1, t=3$ или $k=1, l=1, n=1$, $t=1$. Первьй из этих вариантов дает недопустимое значение $s=5$. Рассмотрим второй вариант. Имеем $P_{g}(x)=\left(x^{18}-1\right)\left(x^{3}-1\right)\left(x^{2}-1\right)(x-1), P_{g^{6}}(x)=\left(x^{3}-1\right)^{6}(x-1)^{6}$; $P_{g^{9}}(x)=\left(x^{2}-1\right)^{8}(x-1)^{8}$.

Элементу $g^{9}$ соответствует набор собственных значений $(1,-1,-1,-1,-1)$. Элементу $g^{2}$ соответствует набор собственньх значений $\left(\zeta_{9}, \zeta_{9}^{3}, \zeta_{9}^{3}, \zeta_{9}^{4}, \zeta_{9}^{7}\right)$. Так как $s=4$, все собственные значения оператора $T(g)$ различны. Поэтому среди них встретятся один корень 6-й степени из 1 и один корень 3 -й степени из 1 . Учитьвая эти два соответствия, получаем, что среди собственных значений $T(g)$ имеется еще три первообразных корня 18 -й степени из 1 . Так как $g^{6}$ соответствует набор $\left(\zeta_{3}, \zeta_{3}, \zeta_{3}, 1,1\right)$, эти три первообразных корня степени 18 из 1 при возведении в 6 степень дают одинаковые значения. Но тогда среди собственных значений оператора $\operatorname{Ad}(g)$ пара $\left(\zeta_{3}, \zeta_{3}^{2}\right)$ встретится 3 раза, что противоречит виду $P_{g}(x)$.

Пусть $\operatorname{ord}(g)=20$. Так как количество единиц среди собственных значений оператоpa $\operatorname{Ad}(g)$ не менее 4 , надо рассмотреть единственньй вариант $P_{g}(x)=\left(x^{20}-1\right)\left(x^{2}-1\right) \times$ $(x-1)^{2}$. Но в этом случае $P_{g^{2}}=\left(x^{10}-1\right)^{2}(x-1)^{4}$, что не допускается.

Остается рассмотреть случаи, когда

1) $P_{g}(x)=\left(x^{13}-1\right)(x-1)^{11}$

2) $P_{g}(x)=\left(x^{17}-1\right)(x-1)^{7}$

3) $P_{g}(x)=\left(x^{19}-1\right)(x-1)^{5}$.

В первом случае среди собственных значений оператора $\operatorname{Ad}(g)$ должно быть 12 единиц, и соответствующий набор собственных значений имеет вид $(a, a, a, b, b)$. Тогда среди неединичных собственных значений оператора будет не более 2 различных, а необходимо получить 12 . Во втором случае среди собственных значений оператора $\operatorname{Ad}(g)$ должно быть 8 единиц, что невозможно. В третьем случае среди собственных значений оператоpa $\operatorname{Ad}(g)$ должно быть 6 единиц, и соответствуюший набор собственных значений имеет вид $(a, a, b, c, d)$. Тог да среди неединичных собственных значений оператора будет не более 12 различных, а необходимо получить 18.

Таким образом, для элементов, порядок которых равен $13,16,17,18,19,20,22,23$, 24, невозможно построить соответствие, удовлетворяющее условию теоремы.

Теорема доказана. 


\section{СПИСОК ЦИТИРОВАННОЙ ЛИТЕРАТУРЫ}

[1] Воскресенскал Г. В. Метацик лические группы и модулярные формы // Матем. заметки. 2000. T. 67. № 2. C. 18-25.

[2] Воскресенская Г. В. Конечные группы и мультипликативные $\eta$-произведения // Вестник СамГУ. 2000. Т. 16. C. $18-25$.

[3] Dummit D., Kisilevsky H., McKay J. Multiplicative products of $\eta$-functions // Contemp. Math. 1985. V. 45. P. 89-98.

[4] Mason G. Finite groups and Hecke operators // Math. Ann. 1989. V. 282. P. 381-409.

[5] Mason G. $M_{24}$ and certain automorphic forms // Contemp. Math. 1985. V. 45. P. 223-244.

[6] Koike M. On McKay's conjecture // Nagoya Math. J. 1984. V. 95. P. 85-89.

[7] Kondo T. Examples of multiplicative $\eta$-products // Sci. Pap. Coll. Arts and Sci. Univ. Tokyo. 1986. V. 35. P. 133-149.

[8] Martin Y., Ken O. Eta-quotients and elliptic curves // Proc. Amer. Math. Soc. 1997. V. 125. № 11. P. 3169-3176.

[9] Gordon B., Sinor S. Multiplicative properties of $\eta$-products // Lecture Notes in Math. V. 1395: Springer-Verlag, 1989. P. 173-200.

[10] Воскресенская Г. В. Модулярные формы и представления групп диэдра // Матем. заметки. 1998. Т. 63. №1. С. 130-133.

[11] Коксетер К. С. М., Мозер У. О. Дж. Порождающие элементы и определяющие соотношения дискретных групп. М.: Наука, 1980.

[12] Воскресенская Г. В. Параболические формы и конечные подгруппы в $S L(5, \mathbb{C}) / /$ Функцион. анализ и его прил. 1995. Т. 29. № 2. С. 1-73.

[13] Воскресенская Г. В. Модулярные формы и регулярные представления групп порядка 24 // Матем. заметки. 1996. Т. 60. №2. С. 292-294.

[14] Voskresenskaya G. V. One special class of modular forms and group representations // J. Théor. Nombres Bordeaux. 1999. V. 11. P. 247-262. 